\title{
ARTÍCULO
}

\section{Investigación ciudadana y prevención de criminalidad en la ciudad de Urbana Illinois}

\author{
Rojas - Álvarez, Jorge*, Wong, Adrian \\ * Estudiante doctoral, Universidad de Illionis en Urbana- Champaign \\ jorger3@illinois.edu \\ ○ Estudiante doctoral, Universidad de Illionis en Urbana- Champaign \\ adrianw3@illinois.edu
}

Este artículo explora cómo el análisis y la visualización de datos de gobierno abierto pueden revelar ciertos desequilibrios en el mantenimiento de la construcción, las formas de redes de comunicación, el reconocimiento de recursos históricos y culturales, y la medición del delito en un distrito de la ciudad de Urbana, Illinois, EE. UU. Este artículo se deriva de un proyecto de investigación centrado en el gobierno de la ciudad de Urbana (Illinois, EE. UU.), el distrito 3 de la ciudad y el trabajo de los estudiantes del seminario de posgrado sobre Datos en Comunidad de la Universidad de Illinois Urbana-Champaign. Un objetivo del proyecto es buscar modelos alternativos para comprender y medir las relaciones entre la policía y la comunidad, especialmente en lo que respecta a las comunidades socioeconómicamente diversas. Como marco teórico-metodológico, el proyecto propone un enfoque participativo de Investigación-Acción en diálogo con el concepto de Nepantla de Gloria Anzaldúa, que sirve como análisis para comprender la investigación ciudadana en esta comunidad. En este artículo, la prevención de crímenes basada en urbanismo ofrece un punto de vista único para considerar las relaciones entre los espacios públicos y la medición del delito en el área de vivienda de interés social de Crystal Lake Townhome del distrito 3 en Urbana City, Illinois, en comparación con otras áreas.

\section{Palabras clave:}

Datos comunitarios, investigación ciudadana, visualizaciones de datos, prevención de crimen basada en urbanismo, Nepantla

\section{INTRODUCCIÓN}


Investigación ciudadana y prevención de criminalidad en la ciudad de Urbana Illinois.

IJESJP

Los datos abiertos como una herramienta para promover diálogo entre comunidades y gobiernos implican grandes retos en la elaboración de argumentos para el desarrollo de planes de mejoramiento que atiendan situaciones problemáticas de dichas comunidades. Para las comunidades y los académicos, los desafíos de utilizar los datos abiertos del gobierno para crear argumentos para reclamar la mejora de los servicios o para obtener más tracción sobre sus problemas han creado conversaciones animadas sobre el uso de la tecnología con fines sociales.

En este caso, se utilizó un proceso de investigación-acción participativa, la realización de entrevistas semiestructuradas, una encuesta y el diseño de visualizaciones basadas en datos del gobierno de la ciudad de Urbana. Se obtuvo una comprensión compartida y gráfica de la problemática de prevención de crímenes basada en el urbanismo (denominado CPTED por sus siglas en inglés), la construcción de comunidades seguras en la ciudad de Urbana y el reconocimiento de lugares de memoria como patrimonio comunitario de la ciudad. Se diseñaron las visualizaciones de datos como objetos provocativos y exploratorios que enfatizan cuestiones de interés, pero también como invitaciones para invertir en el uso de datos y discutir sobre problemas de la comunidad.

El proceso de investigación ciudadana mostrado en este artículo puede entenderse desde el enfoque de Nepantla. Gloria Anzaldúa (2016) describió Nepantla (cruce de fronteras) como experiencias de intermediación y creaciones de poblaciones que desde las fronteras desarrollan formas de entender sus contextos sociales. En este caso, las fronteras cognitivas de los grupos sociales y comunidades involucradas.

El espacio elegido para elaborar este proyecto fue el seminario de posgrado de Datos en Comunidad de la Universidad de Illinois. Este espacio fue liderado por la profesora Anita Say Chan y contó con la participación de estudiantes de posgrado de la Universidad. El seminario se diseñó como catalizador de diálogo entre actores, impulsor del reconocimiento de memoria colectiva de la comunidad base, así como taller de trabajo y experimentación de datos en comunidad. Las problemáticas abordadas por los estudiantes incluyeron la criminalidad y seguridad, recreación y parques, servicio de bomberos y prevención de incendios, así como soporte a productividad y pequeños negocios en la comunidad de base.

La comunidad de base a la que se hará referencia en este artículo son residentes y organizaciones comunitarias del distrito tres (Ward 3) de la ciudad de Urbana. Estos habitantes hacen parte fundamental de un vecindario sociocultural y étnicamente diverso de la ciudad. El vecindario está conformado mayoritariamente por comunidades afroamericanas, afrodescendientes y latinas. En los últimos quince años esa población ha aumentado a más de $25 \%$ de un total de 42.000 personas (American Census Survey, 2019). El distrito tres, ubicado en el noroccidente de la ciudad, fue uno de los distritos sometidos al racismo estructural o redlining (Prochaska, 2016a, 2016b). Como consecuencia de esta desigualdad legalizada, el acceso a servicios, educación,

IJESJP, 2020, V7, n2 
mercados y vivienda fue limitado para los habitantes del distrito. Adicionalmente, el sector cuenta con los más altos índices de criminalidad de la ciudad en los últimos treinta años (City of Urbana, 2019).

El distrito tres sostiene uno de los principales proyectos de vivienda de interés social de la ciudad llamado Crystal Lake Townhome. Este proyecto ha sido un bloque de apartamentos para poblaciones con vulnerabilidad socioeconómica y fue remodelado en la primera década del siglo XXI para actualizar la infraestructura física. Además, se implementaron algunos elementos sugeridos en reportes CPTED que apoyaran la prevención de crimen (Braunfeld, 1991; Weir, 1983). Sin embargo, desde hace varios años se han presentado quejas reiterativas por parte de comunidades del distrito afirmando que los porcentajes en criminalidad también obedecen a selectividad racial por parte del servicio de policía de la ciudad. Por ejemplo, las detenciones de tráfico en la ciudad poseen una disparidad numérica más alta entre minorías raciales dentro de la ciudad (Crime Analysis UPD, 2016; Fields, 2013; Haynes \& Connolly, 2016).

El propósito de este proyecto es mejorar la equidad en la prestación de servicios para comunidades socioeconómicamente diversas de la ciudad de Urbana (Illinois) y especialmente el servicio de policía. Igualmente, el proyecto busca elevar el liderazgo local de comunidades con baja representación en la toma decisiones del gobierno de la ciudad. Se trabajó con residentes y organizaciones de la ciudad. Con estos grupos se diseñó un programa para empoderar miembros de la comunidad que pueden servir como líderes para la ciudad y sus comunidades. Adicionalmente, se busca desarrollar un vínculo entre el conocimiento situado de la comunidad, que articule las voces y necesidades de la esta con las de la ciudad. En ese vínculo se busca plantear problemáticas como la segregación racial en el servicio de policía. Finalmente, se reflexionó sobre la capacidad investigativa de las comunidades gracias a antecedentes de otros trabajos en conjunto con la Universidad de Illinois.

\section{MARCO TEÓRICO-METODOLÓGICO}

\section{Investigación Acción Participativa (IAP) Cíclica}

Ernest Stringer (2013) realiza un aporte metodológico en relación con la intervención organizacional a nivel de comunidades basado en investigación-acción participativa en forma cíclica. Este proceso inicia con la realización de un acercamiento a las situaciones problemáticas de una comunidad en una fase denominada Observar. Posteriormente, se busca que los grupos conformados faciliten el análisis de las causas y desarrollo de las soluciones en una fase denominada Pensar. Luego se elabora un plan y se desarrollan acciones acordadas por la comunidad en la fase denominada Actuar. Culminadas estas etapas puede iniciarse un nuevo 
Investigación ciudadana y prevención de criminalidad en la ciudad de Urbana Illinois.

IJESJP

ciclo o los que requiera la intervención para influir positivamente en las situaciones diagnosticadas.

El presente proyecto se encuentra en una fase de Observar y Pensar porque se ha realizado un trabajo de acercamiento buscando la integración con la comunidad y sus situaciones problemáticas en relación con la prevención del crimen y el reconocimiento de líderes comunitarios del distrito tres. Los siguientes son los aspectos más importantes de esta fase:

- La identificación de los grupos que conforman la comunidad: Es necesario que todos los grupos que conforman la comunidad tengan algún tipo de participación y compromiso dentro de las actividades a realizar. Aunque esta participación no puede ser garantizada, es clave que por lo menos todos los grupos tengan la oportunidad de conocer de qué se trata la posible idea de intervención y que se sientan bienvenidos a unirse cuando lo deseen. Como parte del aprendizaje y reconocimiento de las comunidades participantes, el hecho de identificar y caracterizarse permitirá a los integrantes de las comunidades reconocer la diversidad de los grupos que la integran.

Para este propósito, el seminario de posgrado Datos en Comunidad se diseñó como espacio de diálogo con los grupos que conforman la comunidad. Adicionalmente, el seminario se utilizó como el lugar de construcción de interrogantes sobre los servicios de policía y el reconocimiento de la memoria colectiva de las comunidades. Igualmente, el seminario se diseñó como taller para el uso de datos de gobierno de la ciudad y elaborar argumentos informados sobre situaciones históricas que han afectado dichas comunidades en los temas de seguridad y servicio de policía.

- Debido a que en la comunidad existen integrantes que poseen un mayor reconocimiento por parte de las personas que otras, estos poseen una mayor influencia dentro de algunos grupos. Estas personas denominadas por Stringer (2013) líderes de opinión deben ser incluidos en las primeras etapas del desarrollo de una intervención porque pueden significar un camino para ingresar adecuadamente en la comunidad o un obstáculo para el compromiso en la participación por parte de esta.

Uno de los primeros líderes de opinión es la analista de seguridad del departamento de policía de Urbana y quien es responsable del diseño y análisis de instrumentos estadísticos para la medición de criminalidad en la ciudad. Ella lidera las discusiones sobre el uso de datos por parte de las comunidades y las quejas por excesos sobre poblaciones diversas en la ciudad.

Se realizaron conexiones con empresarios, líderes cívicos y comunitarios, se discutieron las posibilidades de obtener datos más responsables y receptivos, e imaginamos una forma de articular estas formas ocultas de experiencia comunitaria con la ciudad. Por ejemplo, un líder de la una asociación de mejoramiento comunitario en parques actualmente está organizando un grupo interdisciplinario de pensadores y artistas para responder al cambio climático en la Escuela

IJESJP, 2020, V7, n2 
para el Diseño de una Sociedad. Igualmente, otros emprendedores sirven a la comunidad congoleña que vive en Urbana, y han desarrollado una audiencia nacional con sus videos de redes sociales que enseñan a las personas cómo cocinar platos típicos de El Congo. Varios de estos participantes tuvieron intervenciones en clase para elaborar una contextualización de las formas de trabajo de las agencias de policía, bomberos, parques, y trabajo comunitario en la ciudad.

\section{Nepantla: Comunidades investigativas que cruzan fronteras}

También es posible aproximarse a la complejidad de estas comunidades desde sus esfuerzos investigativos. Estas comunidades han generado capacidades para indagar los datos abiertos del gobierno de la ciudad e intervenir en las reuniones públicas de rendición de cuentas y demostrar sus argumentos con la misma información que provee la ciudad. Estos grupos de ciudadanos pueden entenderse desde su intermediación y conformación de puentes entre las comunidades y el gobierno de la ciudad. Con el concepto de Nepantla (Anzaldúa, 2016) se interroga estas experiencias de intermediación como creaciones de poblaciones que desde las fronteras desarrollan formas de entender sus contextos sociales. Dichas creaciones entrelazan su memoria colectiva ancestral con nuevos conocimientos y la necesidad de adaptarse a diversos contextos para desarrollarse. En este sentido, comunidades que han reivindicado sus derechos a través de movimientos sociales traspasan sus fronteras cognitivas para incorporar nuevas formas de diálogo con sus nuevos contextos. Por ejemplo, grupos de estas comunidades en Urbana adquieren conocimientos en ciencias de datos para generar argumentos basados en estadísticas que traducen sus preocupaciones a un lenguaje comprensible para los funcionarios del gobierno. Al trabajar con los mismos datos que el gobierno publica para promover la transparencia de sus acciones, los grupos amplifican sus argumentos. Adicionalmente, al traspasar sus fronteras cognitivas modifican sus identidades y se convierten en intermediarios entre sus comunidades y otros grupos (Anzaldúa, 2009). De esta forma, sus esfuerzos investigativos les convierten en investigadores ciudadanos que preservan y fortalecen sus herencias colectivas mientras incorporan otras nuevas.

El grupo de investigación-acción de la Universidad y el seminario de Datos en Comunidad se desempeña como catalizador para que la Nepantla ocurra con mayor facilidad. El grupo realiza contactos entre los líderes de opinión del distrito 3 y el gobierno de la ciudad. Adicionalmente, cuando es necesario el grupo puede asesorar a los habitantes del vecindario en el desarrollo de capacidades técnicas. Estos contactos plantean preguntas o buscan ofrecer otros puntos de vista sobre las problemáticas de la ciudad con herramientas como las caminatas de datos. Esta es una herramienta etnográfica de aproximación al lugar donde se producen los datos y un acercamiento a las comunidades. A continuación, se describe esta herramienta y su aplicación en el proyecto.

\section{Caminatas de Datos}

IJESJP, 2020, V7, n2 
Investigación ciudadana y prevención de criminalidad en la ciudad de Urbana Illinois.

IJESJP

Una caminata de datos (data walk) es una herramienta etnográfica exploratoria de los lugares de producción de datos sobre la ciudad, sus habitantes y su relación con el entorno (Powell, 2018). Se toman registros fotográficos y escritos de lugares, señales de tránsito, avisos de advertencia o información, infraestructuras físicas que parecen fallidas, abandonadas, funcionando correctamente, etc. Los caminantes inclusive pueden realizar un registro emocional del lugar para dar cuenta qué les inspira, qué les produce algún temor, o por qué toman registro de cierto lugar. Por ejemplo, en una de las caminatas en el parque King del distrito 3, fue sorpresivo observar las barreras físicas que crean los límites entre los parques y su vecindario. Un caminante leía el mensaje de bienvenida en la puerta Norte del parque, este está liderado por la escultura de Preston Jackson Byways to Equality elaborada en acero inoxidable y bronce fundido que recuerda al visitante el movimiento de los años 60 por los derechos sociales civiles en los Estados Unidos. En el lado Oeste del parque no había un límite claro entre las casas y el parque, en el lado este del mismo había una cerca de malla de alambre a lo largo de todo el lado del parque que lo delimita y las casas a su alrededor. En invierno, el color gris oscuro de las cercas contrasta con la nieve blanca. En verano, aparecían pocas plantas en el fondo, así como algunas plantas trepadoras sin ningún tipo de mantenimiento. Con estas observaciones, las caminatas de datos surten el efecto de encontrar intersecciones entre cuestiones o asuntos de interés de los participantes y puntos físicos ricos en posibilidades para activar los datos. La activación de datos en este sentido proporciona la entrada para una reflexión entre el uso de la tecnología y sus impactos sociales.

Además, estos puntos físicos se convierten en puntos de datos que a veces no son procesados por instituciones formales o no están presentes en conjuntos de datos incorporados en la información de planificación del gobierno y la ciudad. En estos ejercicios en los parques del distrito tres, las caminatas de datos proporcionaron rastros de situaciones y contrastes en el mismo vecindario. Por ejemplo, la apertura y los recintos en dos parques que identificaron en ese momento. Por un lado, la apertura dio una impresión de recepción, compartir y atraer. Por otro lado, las barras de metal dieron una impresión de cierre, defensa, prevención y desconfianza. Por supuesto, también implica pensar en la diferencia entre lo público, lo privado y los usos del espacio (P. Cozens \& Love, 2015). También puede interrogarse cómo el parque es un sitio conmemoración y memoria o qué tipos de criminalidad se presentan. Surgen preguntas sobre por qué y cuál podría ser la relación del espacio con los problemas de seguridad y delincuencia de la zona.

A veces, los participantes en caminatas de datos exploran lugares donde nunca antes habían estado. Esta falta de familiaridad les permite contrastar situaciones ocurridas con sus propios barrios, incluso generar nuevas preguntas sobre estos lugares. De todos modos, la actividad es significativa en su experiencia de conocer un lugar, comprender su entorno de una manera diversa y ser conscientes de la producción de datos. En consecuencia, los recorridos de datos se han utilizado como herramientas de participación cívica. Por ejemplo, las pequeñas comunidades

IJESJP, 2020, V7, n2 
en Barcelona han utilizado su comprensión de las brechas en la recopilación de datos del gobierno para comprender, producir datos y negociar con los gobiernos locales sobre las políticas de ruido en sus vecindarios (Smith, Fressoli, \& Thomas, 2014). De esta manera, los recorridos de datos promueven las interacciones entre las comunidades y el gobierno. Por lo tanto, los recorridos de datos proporcionan información, generan preguntas y modifican las formas en que las personas habitan en los espacios públicos.

Aunque las caminatas de datos son predominantemente herramientas de investigación de abajo hacia arriba, el alcance de la participación es amplio y descentraliza los roles de los expertos permitiendo el surgimiento del diálogo entre disciplinas y la negociación de percepciones para producir conocimiento situado (Haraway, 1988). A veces, los grupos de investigación participan junto con funcionarios del gobierno y otros miembros de las instituciones del municipio. Los participantes construyen juntos una versión de sus asuntos de interés. En cualquier discusión sobre inquietudes públicas como la seguridad, el uso de espacios y la criminalidad, el diálogo entre expertos, poblaciones y sus asuntos de interés hacen la diferencia.

\section{Prevención de la delincuencia mediante el urbanismo}

El enfoque de Prevención de la Delincuencia Mediante el Urbanismo (CPTED por sus siglas en inglés) es una aproximación multidisciplinar para disuadir a los delincuentes a alejarse de zonas que han sido intervenidas mediante el diseño de un entorno urbano abierto para la fácil visibilidad y señalización, que muestra la conservación del lugar por parte de los vecinos, y que delimita conscientemente el uso público y privado de las áreas del vecindario. Denominado también la teoría de las ventanas rotas, sugiere que la carencia de diseño urbanístico, mantenimiento de espacios y planeación en el uso de infraestructura física alienta la presencia de criminalidad en dichas zonas de una ciudad (P. M. Cozens, Saville, \& Hillier, 2005).

En este caso, el CPTED se utilizó como herramienta de análisis de la relación entre apropiación de espacios públicos y criminalidad, con el uso de datos abiertos se elaboró un argumento que muestra el desbalance en el mantenimiento de construcciones, formas de redes de comunicación, reconocimiento de recursos históricos y culturales, y la comisión de crimen en la zona de vivienda social Crystal Lake Townhome comparado con otras zonas de la ciudad.

Para esto se utilizó una visualización de datos como una forma didáctica para evidenciar la problemática, hacer uso de los recursos provistos por el gobierno para el uso de datos públicos, y generar una conversación técnica con el departamento de policía. Las visualizaciones tienes un efecto cognitivo más impactante que los datos tabulados y con herramientas de información geográfica proveen un fácil acercamiento a su comprensión por parte de públicos no expertos (Gromme, Ruppert, \& Cakici, 2018).

IJESJP, 2020, V7, n2 


\section{RESULTADOS}

\section{Observaciones de las caminatas de datos}

En las caminatas de datos realizadas, se observaron las barreras físicas que crean los límites entre los parques y su vecindario. La primera exploración fue en Victory Park ubicado en 1000 Green St en Urbana. Mientras el caminante leía el mensaje de bienvenida en la puerta norte, Victory Park fue construido como una conmemoración para los voluntarios que plantaron vegetales en el racionamiento de alimentos al final de la Primera Guerra Mundial. Este parque de cinco acres ofrece servicios para niños, canchas deportivas, y un gran pabellón. El caminante puede encontrar un jardín público en alquiler para fortalecer la vocación pública del parque. Mientras que en el lado Oeste no hay un límite claro entre las casas y el parque, en el lado Este hay una cerca de malla de alambre a lo largo de todo el lado del parque que lo delimita y las casas a su alrededor. En invierno, el color gris oscuro de las cercas contrasta con la nieve blanca. En verano, aparecen pocas plantas en el fondo, así como algunas plantas trepadoras sin ningún tipo de mantenimiento.

La segunda exploración fue en King Park en el distrito tres. Este parque lleva el nombre del Dr. Martin Luther King. Los recorridos por este parque tuvieron un resultado similar en términos de observación de los límites físicos. En este parque de ocho acres en 915 W. Wascher Dr. Urbana, valió la pena notar el contraste entre los límites Sur y Norte. En el límite norte predomina el Wascher Drive, mientras que el lado Sur está delimitado por una cerca de malla de alambre de 5 pies de altura. Contrariamente a Victory Park, en el verano, un buen cuerpo de plantas trepadoras decora la cerca, pero no se observó mantenimiento percibido. Además, el lado Este está liderado por la escultura de Preston Jackson nombrada Byways to Equality hecha de acero inoxidable y bronce fundido que recuerda al visitante el movimiento de los años sesenta por los derechos sociales civiles en los Estados Unidos.

\section{La encuesta sobre el distrito tres de Urbana}

En esta fase del proyecto los funcionarios de la ciudad de Urbana, la profesora Anita Say Chan y estudiantes del seminario de posgrado desarrollaron una encuesta para recopilar datos sobre el distrito tres. La encuesta preguntó información general sobre la trayectoria de los participantes, cuáles planes del gobierno consideran que están funcionando y cuáles no, así como su conocimiento sobre el distrito tres de la ciudad y sobre la zona de Crystal Lake Townhome.

La encuesta arrojó como resultados que algunos de los funcionarios más antiguos de la ciudad que han laborado durante casi treinta años en la institución mostraron muy poco conocimiento sobre el distrito tres. Cuando se presentaron los datos al gobierno de la ciudad, la gran revelación 
para estos funcionarios fue la importancia, pero oculta, de líderes culturales y empresariales que viven y trabajan en el distrito tres.

Respecto a los últimos proyectos de mejoramiento de las condiciones de vida para los habitantes de Crystal Lake Townhome, algunos funcionarios se preguntaron cómo las condiciones de habitabilidad del sector influyen en la seguridad de éste. Ellos identificaron como una pregunta importante la relación entre las necesidades de vida de los habitantes y el mantenimiento del sector. Por ejemplo, identificaron como un proyecto muy positivo el cambio al nuevo Townhome pero llaman la atención que en el largo plazo no parece impulsar mejoras significativas porque los habitantes continúan con los mismos problemas de pobreza y esto no impulsa la inversión en mantenimiento urbano. Algunos encuestados no identificaron que el cuerpo de policía tuviera especial influencia en dichas situaciones porque está dedicado a una labor más reactiva en la respuesta a la criminalidad comparada con vecindarios, asociaciones e inclusive los mismos propietarios de los complejos de viviendas.

\section{Una visualización con datos de gobierno sobre la criminalidad y el ambiente}

Se diseñó una visualización de datos que muestra una superposición entre la comisión de delitos y la falta de mejora de las infraestructuras físicas en el área de Crystal View Townhome en el distrito tres. En términos de datos abiertos del gobierno, este trabajo abordó la complejidad de las estructuras de datos, así como la precariedad de cierta documentación para su fácil consumo para las comunidades. Además, el proceso enfrenta cómo está cambiando la interacción entre las comunidades y las agencias gubernamentales cuando se utilizan argumentos basados en datos. Finalmente, se prestó atención a cómo las comunidades requieren y están dispuestas a adquirir capacidades técnicas para consumir datos y cómo el gobierno y las instituciones educativas deberían apoyar estos procesos para informar mejor su toma de decisión.

Se investigó y amplió la teoría según dos aspectos de infraestructura: lo físico y lo humano. Adicionalmente, en el proceso de esta interacción se apoyó un grupo de funcionarios del gobierno, representantes de la comunidad y estudiantes en el desarrollo de capacidades para el uso de datos abiertos y diseño de visualizaciones basadas en datos.

Desde el punto de vista del uso y producción de datos, se encontró que la producción y consumo de estos está orientada a caracterizar técnicamente los lugares y situaciones de criminalidad, sus tendencias estadísticas y la efectividad en la atención de la comisión de crimen. Esto se demuestra en los datos abiertos del gobierno de la ciudad, que se enfocan en el conteo de arrestos, incidentes, accidentes e inspecciones. Vale la pena decir que este fenómeno no es exclusivo del cuerpo de policía. Por ejemplo, medios de comunicación locales acostumbran a solicitar datos puntuales y contingentes sobre el fenómeno de la criminalidad en la ciudad y muy poco se 
ocupan de realizar informes investigativos detallados de la integralidad del fenómeno de seguridad y criminalidad en la ciudad.

\section{PROPUESTA PARA LA FASE DE ACTUAR}

Se propone que la ciudad de Urbana adopte un programa de becarios como una forma de reconocer la capacidad de investigación y liderazgo dentro de las comunidades. Estos becarios diseñarán programas orientados a la investigación para la diversidad y el espíritu empresarial, la tecnología y las artes, la participación cultural de la comunidad, o diseñarán sus propios proyectos en función de las necesidades que consideren prioritarias.

Aunque muchas instituciones tienen programas de becarios, los gobiernos de las ciudades a menudo no cuentan con estas alternativas a pesar de que los becarios pueden proporcionar valiosa experiencia. Este programa permitirá la investigación práctica a nivel comunitario, ofreciendo acceso abierto a los hallazgos para la ciudad y la comunidad en general.

Se propuso replicar el seminario de posgrado de datos en comunidad en próximos semestres para continuar profundizando en las problemáticas de la ciudad. Igualmente, se piensa fortalecer los contactos con la comunidad a través de nuevos ejercicios, exploraciones de otros puntos del distrito tres, e inclusión de otras agencias del gobierno de la ciudad de Urbana.

\section{CONCLUSIONES}

Se concluyó que el seminario de Datos en Comunidad fue un espacio legítimo de reflexión y encuentro entre funcionarios del gobierno de la ciudad, representantes de la comunidad de la ciudad de Urbana y estudiantes del curso. El seminario catalizó efectivamente el diálogo entre los principales grupos al crear un espacio y un momento de contacto. Adicionalmente, al aportar herramientas como las caminatas de datos, las entrevistas semiestructuradas y la encuesta, el espacio adquirió empatía y legitimidad ante los grupos participantes del proyecto.

El uso del enfoque de prevención de crímenes basado en la apropiación de espacio público permitió establecer puentes de comunicación que nosotros seguimos a llegar a un conocimiento entre los diversos actores del proyecto a través de entrevistas, observaciones de participante y otras maneras de imaginar y acceder a fuentes de datos.

El diseño de visualizaciones generó interrogantes sobre la facilidad de uso de los datos abiertos y la complejidad de su uso para las comunidades. Se concluyó que la visualización es una forma de amplificar la pertinencia del trabajo de las agencias comunitarias y contribuir a la comprensión del delito. El proceso de interacción, la construcción mutua de confianza y el deseo de comprender, así como el argumento basado en la tecnología, han sido significativos para profundizar en más problemas y causas que pueden incluirse en un análisis posterior. Finalmente,

IJESJP, 2020, V7, n2 
este ejercicio sugiere que una conversación abierta sobre datos entre comunidades, agencias gubernamentales e instituciones como la U. de Illinois, debería crear relaciones a más largo plazo e invertir en investigación con un mayor número de comunidades locales, y dedicar recursos en esta fructífera de diálogo.

Este artículo únicamente cubre el trabajo realizado por dos estudiantes del seminario de posgrado de datos de la comunidad. Otros análisis desde el punto de vista del servicio de recreación y parques, servicio de bomberos y prevención de incendios, así como soporte a productividad y pequeños negocios enriquecerían una perspectiva integral de las problemáticas que afrontan las comunidades del distrito tres. Igualmente, dichos análisis podrían complejizar y matizar las reflexiones obtenidas en el presente artículo.

\section{REFERENCIAS}

American Census Survey. (2019). Urbana, IL Population-Census 2010 and 2000 Interactive Map, Demographics, Statistics, Quick Facts. Retrieved September 23, 2019, from CensusViewer.com website: http://censusviewer.com/city/IL/Urbana

Anzaldúa, G. E. (2009). Border Arte: Nepantla, el Lugar de la Frontera. In A. Keating (Ed.), The Gloria Anzaldúa Reader (pp. 176-186). Durham and London: Duke University Press.

Anzaldúa, G. E. (2016). Entrevista con Gloria Anzaldúa por Karin lkas. In C. Valle (Trans.), Borderlands / La Frontera: The New Mestiza (pp. 271-292). Madrid, España: Capitán Swing Libros, S. L.

Braunfeld, K. R. (1991). The impact of crime prevention and defensible space theory and research on planning practice in Illinois (Unpublished Master's thesis, University of Illinois at Urbana-Champaign). Retrieved from https://vufind.carli.illinois.edu/vf-uiu/Record/uiu_3284212

City of Urbana. (2019). Police Incidents Since 1988. Retrieved from Open Data Portal website: https://data.urbanaillinois.us/Police/Police-Incidents-Since-1988/uj4k-8xe8

Cozens, P., \& Love, T. (2015). A Review and Current Status of Crime Prevention through Environmental Design (CPTED). Journal of Planning Literature, 30(4), 393-412. https://doi.org/10.1177/0885412215595440 
Investigación ciudadana y prevención de criminalidad en la ciudad de Urbana Illinois.

IJESJP

Cozens, P. M., Saville, G., \& Hillier, D. (2005). Crime prevention through environmental design (CPTED): A review and modern bibliography. Property Management, 23(5), 328-356. https://doi.org/10.1108/02637470510631483

Crime Analysis UPD. (2016). Report to City Council Concerning Traffic Stop Data (p. 14). Retrieved from Urbana Police Department website: https://www.urbanaillinois.us/sites/default/files/attachments/Urbana_Police_\%20Departm ent_Report_to_City_Council_\%20Concering_Traffic_Stop_Data_\%200CT_\%202016_1. pdf

Fields, B. (2013, October 7). Six Community Organizations Ask Urbana Council for a Study Session on Racial Disparities in Police Stops | The Public i [Independent Media Center]. Retrieved from The Public «i» website: http://publici.ucimc.org/2013/11/six-community-organizations-ask-urbana-council-for-a-s tudy-session-on-racial-disparities-in-police-stops/

Gromme, F., Ruppert, E., \& Cakici, B. (2018). Data scientists: A new faction of the transnational field of statistics. In H. Knox \& D. Nafus (Eds.), Ethnography for a data-saturated world (pp. 33-61). Manchester, England: Manchester University Press.

Haraway, D. (1988). Situated Knowledges: The Science Question in Feminism and the Privilege of Partial Perspective. Feminist Studies, 14(3), 575-599. https://doi.org/10.2307/3178066

Haynes, M., \& Connolly, P. (2016). Overview of the Urbana Police Department's Report to City Council concerning Traffic Stop Data (p. 14). Retrieved from Urbana Police Department website:

https://www.urbanaillinois.us/sites/default/files/attachments/Overview_\%20of_Urbana_P olice_\%20Department_Report_to_City_Council_\%20Concering_Traffic_Stop_Data_\%2 0OCT_\%202016.pdf

Powell, A. (2018). The data walkshop and radical bottom-up data knowledge. In H. Knox \& D. Nafus (Eds.), Ethnography for a data-saturated world (pp. 212-231). Manchester, England: Manchester University Press.

Prochaska, N. (2016a, January). How Champaign's Segregated North End was Created 1940 1960 [Independent Media Center]. Retrieved from The Public «i» website: http://publici.ucimc.org/2016/01/how-champaigns-segregated-north-end-was-created-194 $0-1960 /$

Prochaska, N. (2016b, March). "Old" Urban Renewal in Champaign-Urbana, 1960-1969 [Independent Media Center]. Retrieved from The Public «i» website: http://publici.ucimc.org/2016/03/old-urban-renewal-in-champaign-urbana-1960-1969/ 
Smith, A., Fressoli, M., \& Thomas, H. (2014). Grassroots innovation movements: Challenges and contributions. Journal of Cleaner Production, 63, 114-124. https://doi.org/10.1016/j.jclepro.2012.12.025

Stringer, E. T. (2013). Action Research (4th ed.). Thousand Oaks, CA: SAGE Publications.

Weir, M. L. (1983). Crime in Urbana, Illinois: A comparative study of environmental factors influencing the incidence of crime in public housing (Unpublished Master's thesis, University of Illinois at Urbana-Champaign). Retrieved from https://vufind.carli.illinois.edu/all/vf-uiu/Record/2123837/Description 